\title{
Thunderstorm-resistance Planning of Overhead Distribution Line
}

\author{
Yilin Zhong ${ }^{1, a}$, Chong Ma ${ }^{1, b}$, Zefu Jiang ${ }^{1, \mathrm{c}}$, Kaigui $\mathrm{Xie}^{2, \mathrm{~d}}$, Bo Hu${ }^{2, ~ e}$, Lei Yin ${ }^{2, \mathrm{f}}$, \\ Yanlin $\mathrm{Li}^{2, g}$, Changlin $\mathrm{Li}^{2, \mathrm{~h}}$ \\ ${ }^{1}$ Power Grid Planning Research Center, Guizhou Power Grid Corporation, Guiyang 550002, China; \\ ${ }^{2}$ State Key Laboratory of Power Transmission Equipment \& System Safety and New Technology, \\ Chongqing University, Chongqing 400044, China.

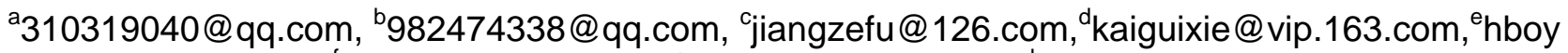 \\ 8361@163.com, ${ }^{\mathrm{y}} \mathrm{y}$ inlei817@163.com, ${ }^{\mathrm{g}} 318797998 @ q q . c o m,{ }^{\mathrm{h}}$ lichanglin2012@foxmail.com
}

Keywords: thunderstorm weather, overhead distribution line, thunderstorm-resistance planning.

\begin{abstract}
Overhead distribution line is easy to suffer lightning trip upon strong thunderstorm due to its low design insulation level, which will reduce reliability of power distribution system. Considering the random annual changes of thunderstorm characteristic parameters, a long-term reliability model of overhead distribution line against thunderstorm disaster was established for strong thunderstorm regions based on the probability distribution model of thunderstorm characteristic parameters. This model takes insulator type and installation interval of arrester as the decision variables and views the cost of power distribution network in the whole life cycle as the objective function. The optimal thunderstorm-resistance planning of overhead distribution line was solved by using the genetic algorithm. The established model was used to make a thunderstorm-resistance planning of the RBTS-BUS2 system. Results showed that based on this model, grid planners could comprehend lightning strike risk level of the power distribution network according to intensity of regional thunderstorm characteristic parameters, and could make reliable and economical planning scheme of insulator type and arrester interval.
\end{abstract}

\section{Introduction}

Overhead distribution line exposed in the natural environment is characteristic of enormous quantity and large-scale coverage, which makes it vulnerable. The power distribution network often will be destroyed in severe weather. Lighting protection design methods of power distribution network mainly include: (1) improve thunderstorm tolerance and insulation characteristic of distribution line; (2) install arc-guiding and arc-extinguishing devices; (3) build overhead ground wire or install arrester; (4) lower ground resistance. Such constant value design ${ }^{[1]}$ is easy to operate, but couldn't reflect reliability and economical efficiency of the design comprehensively.

Reference [2] studied the disaster-resistance model of grid planning and put forward a disaster-resistance grid planning design considering typical natural disasters. It established a high-efficiency mathematical model of disaster-resistance grid planning by introducing in nonlinear method of separated inequality constraint and embedded N-1 constraint elimination model, thus realizing the refining decision-making on investment for disaster resistance of power grid. Reference [1] made a statistical analysis on effect of wind disaster, lightning disaster and flood damages on safe operation of the medium voltage distribution network in Maoming where has severe weather conditions. It proposed not only a medium voltage distribution network planning method considering wind disaster, lightning disaster and flood damages, but also investment programs for different disaster-resistance grid planning. Reference [3] proposed different grid planning methods against lightning fault by analyzing thunder intensity, environmental factors and characteristics of power distribution network state. Reference [4] pointed out that grid planning considering disastrous weather must quantify reliability indexes and view fault losses caused by severe weather as one of the objective functions. With consideration to effect of region characteristics on various index weights, Reference [5] enlisted risk indicator of line fault caused by severe weather into the comprehensive evaluation system and proposed the grid planning that 
involves characteristics of the study area and resistance to severe weather. Combining with wind disaster in Guangdong Province, Reference [6] estimated engineering cost considering effect of wind disaster and proposed different medium voltage distribution network design programs with high wind and disaster tolerances.

Based on above literature review, existing researches on disaster resistance of power distribution network mainly focus on wind disaster and ice disaster. Few researches on thunderstorm-resistance planning of overhead distribution line have been reported yet. However, medium voltage distribution network has "congenitally deficient” thunder resistance and low design insulation level, thus difficult to withstand direct lightning and inductive thunder. Therefore, it is urgent to study the thunderstorm-resistance planning of overhead distribution line. Secondly, existing researches mainly concentrated on disaster-resistance frame structure of electrical power system, but didn't involve selection of overhead distribution line element. They are difficult to apply grid planning method into engineering practice.

Therefore, to achieve thunderstorm resistance of overhead distribution line that considering optimal resource allocation, economical efficiency and reliability, this paper attached high attentions on the optimal insulator type selection and the optimal planning of arrester interval for overhead distribution line.

In this paper, the probability distribution model of thunderstorm characteristic parameters was established with non-parameter kernel density estimation method. Based on this model, a reliable and economical thunderstorm-resistance planning model of overhead distribution line was proposed. In this planning model, full life cycle cost of power distribution network including investment cost, operating maintenance cost and outage cost is the objective function, and insulator type and arrester interval are decision variables. This planning model was solved by the genetic algorithm to select the optimal insulator type and plan the optimal arrester interval for overhead distribution line in regions with strong thunderstorm.

\section{Reliability modeling of overhead distribution line}

\subsection{Random probability distribution modeling of annual thunderstorm characteristic parameters.}

Annual thunderstorm characteristic parameters mainly include lightning days and lightning current amplitude. The service cycle of overhead distribution line lasts decades of years. The overhead distribution line shall be designed strong enough to resist different-intensity of thunderstorms in the service cycle. Therefore, a probability model of historical thunderstorm parameters shall be established during grid planning design.

(1) Probability model of lightning days

Lightning days ${ }^{[7]}$ refer to days having thunder and lightning in one year. It doesn't obey any specific distribution and could only establish a model through non-parameter estimation methods. Among so many non-parameter estimation methods, empirical distribution function (EDF) is a very important one and has been widely used in statistics and engineering field ${ }^{[9]}$. It gets one approximation of practical distribution function through frequency-based probability estimation of samples. Therefore, this paper used EDF to establish the probability distribution model of lightning days.

Suppose $X=\left\{X_{1}, X_{2}, X_{3}, \ldots X_{n}\right\}$ is a group of sample data of historical lightning days. If probability of occurrence of each sample is $1 / n$, the empirical distribution probability density function of lightning days could be expressed as ${ }^{[17]}$ :

$$
\hat{F}(x)=\frac{\sum_{i=1}^{n} S\left(X_{i} \leq x\right)}{n}
$$

where

$$
S\left(X_{i} \leq x\right)=\left\{\begin{array}{l}
1 \text { if } X_{i} \leq x \\
0 \text { if } X_{i}>x
\end{array}\right.
$$


where $X_{i}$ is sample size of lightning days and $S\left(X_{i} \leq x\right)$ is an indicator function.

(2) Probability model of lightning current amplitude

lightning current refers to current that flow into the ground through the stroke objects by direct lightning. lightning current amplitude is a random variable and is closely related with natural conditions as well as meteorological conditions. Its probability distribution can only be estimated accurately from abundant measured data. Currently, the probability distribution model of lightning stroke circuit in common regions which is recommended by Chinese regulations ${ }^{[7]}$ is:

$$
P(I)=10^{\frac{-I}{88}}
$$

where $I$ is lightning current amplitude (unit: kA).

\subsection{Modeling for lightning trip of overhead distribution line}

The striking distance model of medium voltage overhead distribution line without overhead ground wire protection[10] (or called as the electric geometry model, EGM) is shown in Fig.1.

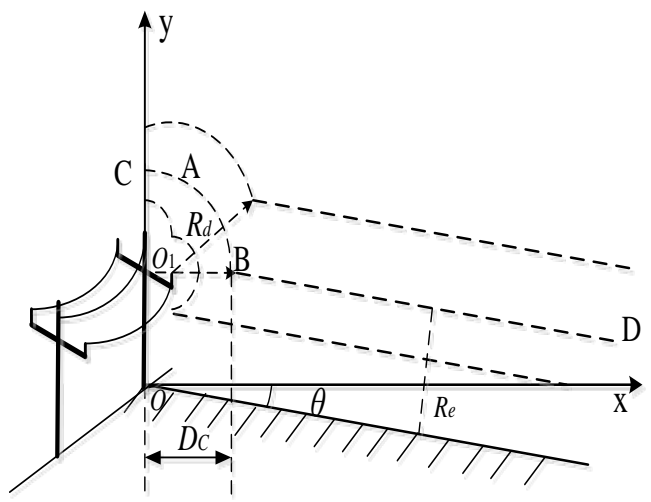

Fig.1 The electrical geometry model of power distribution lines without overhead ground wire

In Fig.1, arc CAB is the exposure arc of wires; arc BD is the exposure arc of the earth; $R_{d}$ is the striking distance to the wires; $R e$ is striking distance to the ground; $\theta$ is inclination of the ground.

$R_{d}$ is related with both lightning current amplitude and wire height. Its calculation formula proposed by Eirksson is:

$$
R_{d}=0.67 h^{0.6} I^{0.74}
$$

where $R_{d}$ is striking distance to wires (unit: $\mathrm{m}$ ) and $h$ is the mean height of wires (unit: $\mathrm{m}$ ).

The calculation method of $R_{e}$ recommended by the IEEE working team is:

$$
\left.R_{e}=(0.168 \ln (43-h)+0.36)\right) \times R_{d}
$$

The lightning current when the wire height is equivalent to the height of intersection of $R_{d}$ and $R_{e}$ (point $\mathrm{B}$ ) is the critical lightning current $I_{0}$ :

$$
I_{0}=\left(\frac{h^{0.4} \times \cos \theta}{0.67 \times(0.168 \ln (43-h)-\sin \theta+0.36)}\right)^{1 / 0.74}
$$

The unilateral lightning attraction scope of overhead distribution line (D) recommended by China's electric power industry is calculated through:

$$
D=\int_{0}^{I_{0}} R_{d} p(I) d I+\int_{I_{0}}^{+\infty} x_{k} p(I) d I
$$

where $x_{k}$ is the $\mathrm{x}$-coordinate value of point $\mathrm{B}$ (unit: $\mathrm{m}$ ) and $p(I)$ is the probability density function of lightning current amplitude:

$$
p(I)=\frac{\ln 10}{88} 10^{\frac{-I}{88}}
$$

The trip-out rates of overhead distribution line caused by direct lightning and inductive thunder are expressed as equation (9) and (10), respectively.

$$
T R_{d}=N_{g} \times \eta \times \frac{b+2 D}{10} \times 10^{-I_{e} / 88}
$$




$$
T R_{i}=0.2 \times N_{g} \times \eta \times \int_{D}^{+\infty} 10^{-I_{e}(s) / 88} d s
$$

where $N_{g}$ ( $/ \mathrm{km}^{2}$ per year) is the cloud-to-ground lightning, $b(\mathrm{~m})$ is the projection width of the top wire on the ground, $I_{e}$ is lightning withstand level ${ }^{[7]}, s$ is the distance from the striking point to the circuit, and $\eta$ is the arc over rate. According to criteria of China's power industry, arc over rate of the over voltage protection and insulation coordination (DLT520-1997) in the AC electric installation could be expressed as:

$$
\eta=\left(4.5 E^{0.75}-14\right) \times 10^{-2}
$$

where $E(\mathrm{kV} / \mathrm{m})$ is the mean running voltage gradient of insulator string.

Combining equation (9) and (10), the lightning trip-out rate of overhead distribution line is:

$$
T R_{l}=T R_{d}+T R_{i}
$$

\subsection{Modeling for lightning trip-out rate of overhead distribution line}

Most insulation flashover failures of overhead distribution line caused by lightning stroke are instantaneous ones which could be cleared by the automatic circuit recloser ${ }^{[11]}$. Neglecting sensitive load voltage sag caused by the automatic circuit recloser, these failures generally won't cause power cut to users. However, some failures couldn't be cleared by the automatic circuit recloser and may cause forced outage of circuit. These are called as permanent faults. Reference [12] pointed out that the occurrence rate of permanent circuit fault under thunderstorm weather is approximately equivalent to failure rate of overhead distribution line.

To simplify analysis, this paper assumed that the forced outage proportionality coefficient of lightning stroke $\alpha=0.195$. It is important to note that $\alpha$ may be different in different regions. It could be calculated through local historical forced outage statistics caused by lightning stroke.

According to equation (9), the failure rate $\left(\lambda_{\text {lighting }}\right)$ of overhead distribution line under $x_{i}$ and $I_{j}$ is:

$$
\lambda_{\text {lighting }}\left(x_{i}, I_{j}\right)=\alpha \times T R_{l}\left(x_{i}, I_{j}\right)
$$

Therefore, the comprehensive mean failure rate of lightning-induced fault and normal fault mentioned in Reference [13] could be calculated from:

$$
\lambda_{1+n}=\frac{T_{\text {lightning }}}{T_{\text {year }}}\left(\lambda_{\text {lightning }}+\lambda_{\text {normal }}\right)+\frac{T_{\text {normal }}}{T_{\text {year }}} \lambda_{\text {normal }}
$$

where $T_{\text {lightning }}, T_{\text {normal }}$ and $T_{\text {year }}$ are lightning time, normal time and total time of the overhead distribution line; $\lambda_{l+n}, \lambda_{\text {lightning }}$ and $\lambda_{\text {normal }}$ are the comprehensive failure rate, lightning-induced failure rate and failure rate under normal weather of the overhead distribution line.

MTTR of overhead distribution line under thunderstorm weather is calculated as:

$$
r_{l+n}=\frac{T_{\text {lightning }}}{T_{\text {year }}} r_{\text {lightning }}+\frac{T_{\text {normal }}}{T_{\text {year }}} r_{\text {normal }}
$$

where $r_{l+n}, r_{\text {lightning }}$ and $r_{\text {normal }}$ are MTTR of overhead distribution line, MTTR under thunderstorm weather and MTTR of normal faults.

\section{Distribution network planning model considering thunderstorm disaster}

\subsection{Decision variables}

Traditional distribution network planning model adopts same thunder protection measures to lines in the same region, but doesn't consider effect of ground angle of pole of overhead distribution line on lightning fault of the distribution network. To improve efficiency of distribution network planning, different thunder protection measures shall be applied to different-oriented distribution lines in the same region.

Insulator is the favorite insulation material in overhead distribution line structure. Different types of insulator has different insulation intensities. It can be known from equation (9) and (10) that increasing 50\% discharge voltage could reduce the direct lightning flashover probability of lines significantly. Therefore, this paper took the insulator distribution mode with the highest reliability 
and economical efficiency as one of the decision variables of the proposed planning model.

Generally, arrester in the power network is used to protect transformer and voltage regulator. It controls voltage of the protected distribution equipments within a certain range by guiding lightning current into the ground, thus protecting the equipments insulated. In countries around the whole world, arrester is widely used in overhead distribution line to improve thunderstorm tolerance $\mathrm{e}^{[14][15][16]}$. In Japan, there are abundant researches on arrester installation method and arrester is widely applied, which reduces lightning accidents of lines greatly.

Table 1 shows lightning protection effect of different arrester installation intervals provided in Reference [14].

Tab.1 Comparison of lightning protection effect under different installation intervals of arrester

\begin{tabular}{ccc}
\hline Installation interval of arrester & $\begin{array}{c}\text { Reduction of direct } \\
\text { lightning trip-out } \\
\text { rate }\end{array}$ & $\begin{array}{c}\text { Reduction of inductive } \\
\text { lightning trip-out rate }\end{array}$ \\
\hline 1 & $0 \%$ & $100 \%$ \\
2 & $0 \%$ & $96 \%$ \\
3 & $0 \%$ & $95 \%$ \\
4 & $0 \%$ & $70 \%$ \\
5 & $0 \%$ & $58 \%$ \\
6 & $0 \%$ & $47 \%$ \\
No arrester & $0 \%$ & $0 \%$ \\
\hline
\end{tabular}

It can be seen from Table 1 that installation of arrester could lower inductive lightning trip-out rate significantly. According to operating experiences, arrester could protect overhead distribution line from lightning flashover fault completely. However, it is invalid to reduce circuit fault caused by direct lightning. Moreover, shortening installation interval of arrester could reduce inductive lightning trip-out rate of overhead distribution line greatly. Therefore, this paper took the installation interval of arrester with the highest reliability and economical efficiency as one of the decision variables of the proposed planning model.

\subsection{Objective function and constraints}

Thunderstorm-resistance planning of overhead distribution line will surely increase the construction cost and investment, but lightning failures will be decreased, which will reduce outage cost accordingly. Therefore, the proposed planning model took the minimum life cycle cost of power distribution network $(f)$ which includes investment cost $\left(f_{1}\right)$, operating maintenance cost $\left(f_{2}\right)$ and outage cost $\left(f_{3}\right)$ as the objective function:

$$
\min f=f_{1}+f_{2}+f_{3}
$$

In this paper, installation interval of arrester $\left(n_{p}\right)$ was viewed as one constraint:

$0 \leq n_{p} \leq 6$

where $n_{p}$ is an integer. If $n_{p}=0$, no arrester is installed on the overhead distribution line.

\subsubsection{Investment cost}

In thunderstorm-resistance planning, the investment cost of power distribution network includes installation costs of insulators and arrester as well as equipment maintenance cost except conventional construction cost.

$$
\begin{aligned}
f_{1} & =A_{p}\left(r_{0}, n\right)\left(\left(6 n_{j} m_{i j}+n_{b} m_{b}\right)+S n_{r}\right) \\
n_{j} & =\frac{S}{l} \\
n_{b} & =\operatorname{int}\left(\frac{S}{n_{p} \times l}\right)+1 \text { or } n_{p}=0, n_{b}=0 \\
A_{p} & =\frac{r_{0}\left(1+r_{0}\right)^{m}}{\left(1+r_{0}\right)^{m}-1}
\end{aligned}
$$


where $A_{p}\left(r_{0}, n\right)$ is funds recovering coefficient ( $r_{0}$ is capital discount rate and $m$ is depreciation year of equipment); $S$ is length of distribution line; $l$ is line span, which is fixed $55 \mathrm{~m}$ in this paper; $n_{j}$ is number of installed insulators; $m_{i j}$ is the unit price of the $i^{\text {th }}$ insulator; $n_{b}$ is number of installed arrester; $m_{b}$ is the unit price of arrester; $n_{p}$ is the configuration of arrester: $n_{p} \in[0,6] ; n_{r}$ is price of line per unit length.

It should be noted that the $10 \mathrm{kV}$ overhead line in this paper is 3-phase one-way line and there are 2insulators in the insulator chain. Arrester is installed on all 3-phase line and 3 arrester are divided into one group.

\subsubsection{Operating maintenance cost}

After finished line construction, lines, insulators and arrester shall be maintained regularly. Therefore, operating maintenance cost covers operation cost and maintenance cost of equipments. The operating maintenance cost of overhead distribution line could be expressed as:

$$
f_{2}=S m_{c 1}+6 n_{j} m_{c 2}+n_{b} m_{c 3}
$$

where $m_{c 1}$ is annual maintenance cost per unit length of distribution lines; $m_{c 2}$ is annual maintenance cost of every insulator; and $m_{c 3}$ is annual maintenance cost of every group of arrester.

\subsubsection{Outage cost}

Outage cost includes power failure cost caused by thunderstorm and other weather conditions.

$$
f_{3}=\sum_{i=1}^{n} U_{(l+n) i} p_{i} m_{i}
$$

where $U_{(l+n) i}$ is the mean outage time of failure load point $i$ of power distribution network element under thunderstorm and normal weather conditions; $p_{i}$ is load carried by the load point $i$; and $m_{i}$ is outage cost per $\mathrm{kWh}$ of the load point.

\section{Solving algorithm of thunderstorm-resistance planning model of overhead distribution line}

Genetic algorithm (GA) ${ }^{[17][18]}$ has characteristics of robustness, easy-to-operation and implicit parallel processing. It selects the superior ad eliminates the inferior based on individual reproduction, crossover and mutation during population evolution, thus approaching to the optimal solution gradually. The optimization process of GA is mainly realized through individual selection, crossover and mutation operators.

\subsection{Chromosome codification}

Four types of insulators were considered in this paper. Arrester distribution mode is shown in Table 1, including 7 types. Therefore, insulator type could be expressed by 2-bit binary number and arrester distribution mode could be represented by 3-bit binary number (Fig.2).

\begin{tabular}{|l|l|l|l|l|}
\hline$\alpha_{1}$ & $\alpha_{2}$ & $\beta_{1}$ & $\beta_{2}$ & $\beta_{3}$ \\
\hline
\end{tabular}

Fig.2 Chromosome codification of this paper

\subsection{Fitness function}

The objective function in this paper is the minimum life cycle cost of the distribution network. Then, the fitness function can be set as:

$$
\text { fitness }(x)=f_{\max }-f(x)
$$

where $f_{\max }$ is the maximum objective function in every generation and $f(x)$ is the life cycle cost of the distribution network corresponding to chromosome $x$. Therefore, individual have higher fitness value is more probably to be retained.

\subsection{Genetic operators}

(1) Selection operator

Set the population size as $n$ and fitness of the individual $i$ in the population as $f_{i}$. Then, the probability of $i$ to be chosen can be expressed as the proportion of its fitness in the total fitness of all individuals: 


$$
P_{i}=\frac{f_{i}}{\sum_{i=1}^{n} f_{j}}
$$

(2) Crossover and mutation operators

The calculation formula of self-adaptive crossover operator is:

$$
P_{c g}=P_{c \max }-\omega\left(P_{c \max }-P_{c \min }\right)
$$

where $P_{c g}$ is the crossover rate of the $g^{\text {th }}$ generation; $P_{c \max }$ is the upper limit of a given crossover rate; $P_{c \text { min }}$ is the lower limit of a given crossover rate; and $\omega$ is fitness coefficient, which could be calculated from:

$$
\omega=\frac{D_{g}}{\max _{1 \leq r \leq g} D_{r}}
$$

where $D_{r}$ is the standard deviation of fitness of the $r^{\text {th }}$ chromosome.

The calculation formula of self-adaptive mutation rate is:

$$
P_{m g}=P_{m \max }-\omega\left(P_{m \max }-P_{m \text { min }}\right)
$$

where $P_{m g}$ is the mutation rate of the $g^{\text {th }}$ generation; $P_{\text {mmax }}$ and $P_{m \text { min }}$ are the upper and lower limit of a given mutation rate.

\section{Algorithm flowchart}

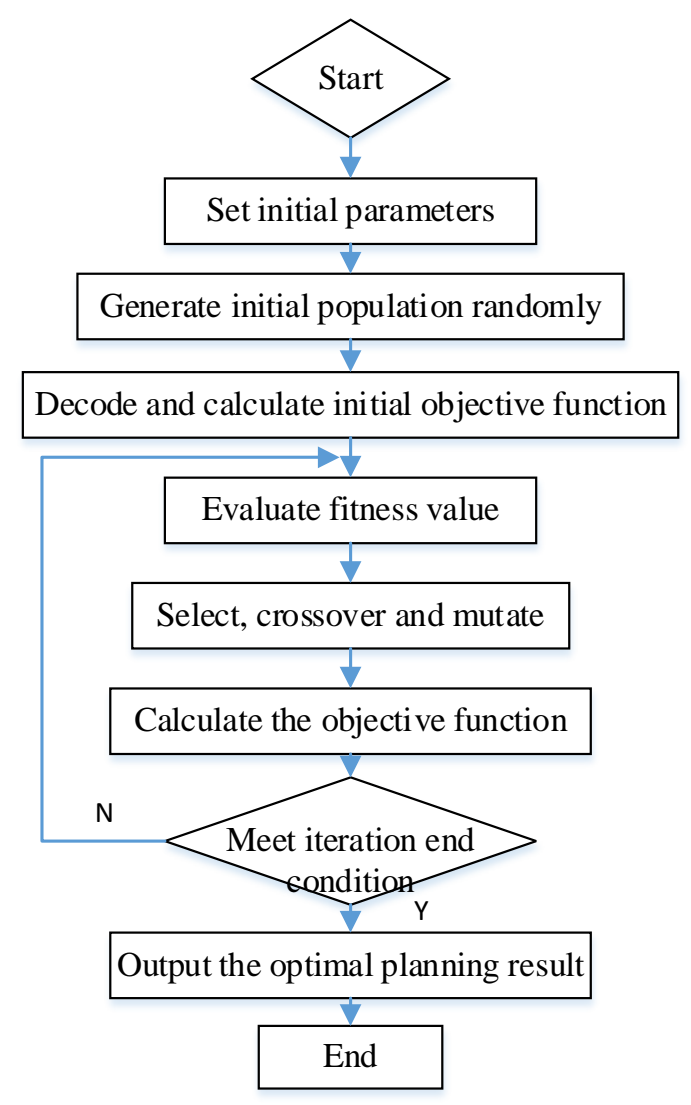

Fig.3 Algorithm flowchart of insulators and surge arrester layout optimization on medium voltage overhead distribution line

The planning optimization process of overhead distribution line considering thunderstorm disaster is shown in Fig.3. Steps are introduced as follows:

Step1: Set input parameters: population size, upper and lower limits of crossover rate and mutation rate, selection rate, iterations.

Step2: Generate the initial population randomly.

Step3: Decode chromosome and calculate objective function of the initial population. 
Step4: Calculate fitness value of individuals.

Step5: Select, crossover and mutate chromosome.

Step6: Calculate the objective function of the new individual.

Step7: Judge whether meets the iteration end condition. If yes, output the optimization result; otherwise, turn to Step4.

\section{Case study}

Thunderstorm-resistance planning of feeder F2 and feeder F4 in the RBTS-BUS2 was implemented using the GA in Chapter 3. The ground elevations of feeder F2 and feeder F4 are $0^{\circ}$ and $45^{\circ}$, respectively. This system has a total of 9 load points, with an average load of $5.54 \mathrm{MW}$. The system structure is shown in Fig.4.8. Reliability parameters and electric parameters are introduced in Reference [19].

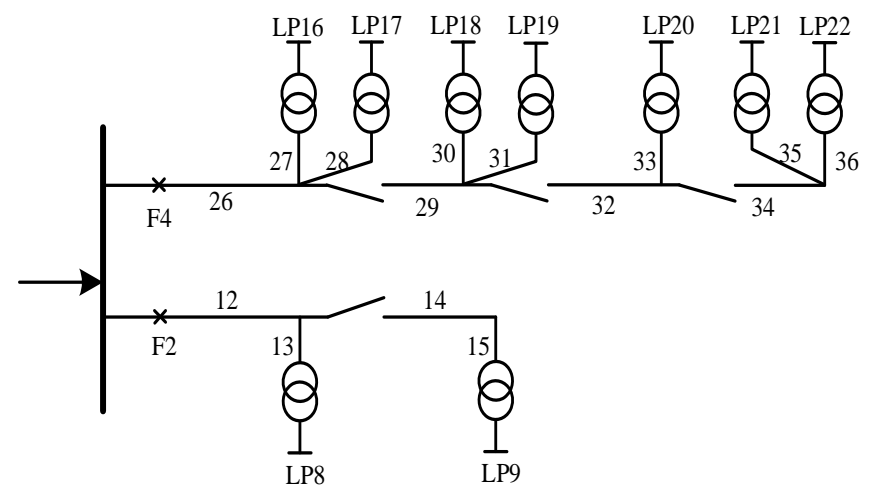

Fig.4Feeder F2 and feeder F4 of RBTS-BUS2 test system

Prices of different insulators are exhibited in Table 2.

Tab.2 Price of different insulators

\begin{tabular}{cc}
\hline Type of insulator & Price (RMB/pc) \\
\hline Pin insulator & 20 \\
Composite insulator & 40 \\
Porcelain-arm insulator & 50 \\
Glass insulator & 30
\end{tabular}

Additionally, the price of arrester is 150RMB. According to Reference [20], maintenance cost per $1 \mathrm{~km}$ is $2,000 \mathrm{RMB}$ /year and operating cost per $1 \mathrm{~km}$ is $2,000 \mathrm{RMB} /$ year. The planning cycle is 15 years. The average price of element per unit length of line is 500RMB and outage cost per unit is 4RMB, with a discount rate of $10 \%$.

\subsection{Probability distribution analysis of annual thunderstorm characteristic parameters}

In this part, inter-annual distribution characteristics of lightning days were analyzed based on the daily observed data of thunderstorms in Danzhou, Hainan form 1953-2012 ${ }^{[21][22]}$.

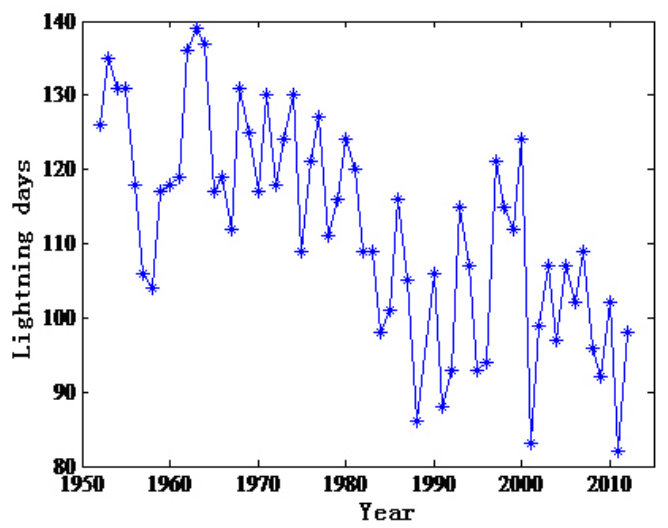

Fig.5 Inter-annual variation of lightning days in Danzhou Hainan

The probability distribution of lightning days in Danzhou could be known through empirical 
distribution estimation (Fig.6).

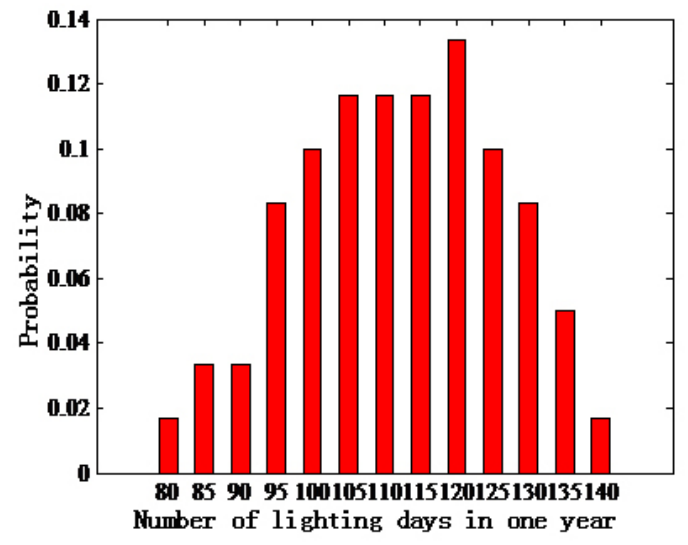

Fig.6 Probability density distribution of thunderstorm

The probability density distribution function of lightning current amplitude is shown in Fig.7.

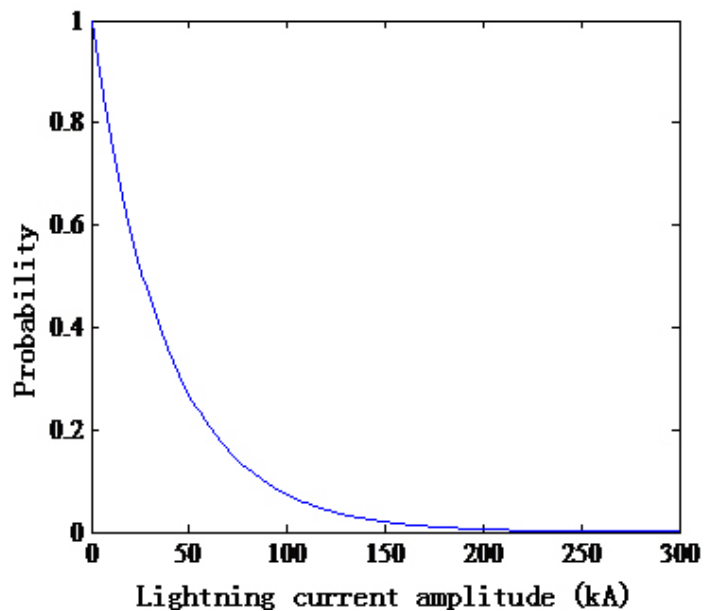

Fig.7 Probability density distribution of lightning current amplitude

\subsection{Optimization thunderstorm-resistance planning of overhead distribution line}

\subsubsection{Optimization results}

RBTS-BUS2 system was optimized to resist thunderstorm according to the proposed method in Chapter 4. Optimization results are presented in Table 3.

Tab.3 Optimal plan of insulator and installation interval of arrester on overhead distribution lines

\begin{tabular}{ccc}
\hline Type of insulator & $\begin{array}{c}\text { Installation interval of } \\
\text { arrester }\end{array}$ & LCC (10,000RMB) \\
\hline Pin insulator & $\begin{array}{c}\text { Arrester is installed on all } \\
\text { towers }\end{array}$ & 45.776
\end{tabular}

According to Table 3, the minimum life cycle of power distribution network is achieved by using pin insulator and installing arrester on all towers. It can be seen from Table 1 that smaller installation interval of arrester causes fewer inductive lightning faults of overhead distribution lines and smaller outage cost, thus enabling the power distribution system to reach balanced reliability and economical efficiency. Therefore, installing arrester on overhead distribution lines has higher cost performance than increasing insulation level of lines.

At present, pin insulator and one group of arrester at the end of the overhead distribution line is the universal design scheme of power distribution network ${ }^{[70]}$. Table 4 is the cost comparison between conventional planning and the proposed planning. 
Tab.5 Comparison of plan schemes

\begin{tabular}{ccc}
\hline Item & $\begin{array}{c}\text { Pin insulator but no } \\
\text { arrester }\end{array}$ & $\begin{array}{c}\text { Pin insulator and } \\
\text { arrester on all lines }\end{array}$ \\
\hline $\begin{array}{c}\text { Investment cost } \\
\text { (10,000 RMB) }\end{array}$ & 23.398 & 23.585 \\
Operating maintenance cost & & \\
(10,000 RMB) & 1.245 & 1.247 \\
Outage cost (10,000 RMB) & 24.744 & 20.935 \\
LCC uniform annual & & \\
value(10,000 RMB) & 49.388 & 45.776 \\
\hline
\end{tabular}

Compared to the planning using pin insulator but no arrester, the planning using pin insulator and arrester on all lines has poorer economical efficiency, but its outage cost reduces by 38,090RMB. The power distribution network considering both economical efficiency and reliability has lower LCC.

\subsubsection{Analysis of influencing factors}

Economical parameters that influence LCC of power distribution network include discount rate and unit outage cost. With the change of electricity market, economical parameters will change accordingly.

(1) Effect of discount rate

Tab.6 Effect of discount rate on distribution system planning

\begin{tabular}{cccc}
\hline Discount rate & $\begin{array}{c}\text { Type of } \\
\text { insulator }\end{array}$ & Installation interval of arrester & $\begin{array}{c}\text { LCC } \\
(10,000 \\
\text { RMB })\end{array}$ \\
\hline $8 \%$ & Pin insulator & Arrester is installed on all towers. & 43.149 \\
$9 \%$ & Pin insulator & Arrester is installed on all towers. & 44.446 \\
$10 \%$ & Pin insulator & Arrester is installed on all towers. & 45.776 \\
$11 \%$ & Pin insulator & Arrester is installed on all towers. & 47.138 \\
$12 \%$ & Pin insulator & Arrester is installed on all towers. & 48.507 \\
\hline
\end{tabular}

It can be seen from Table 6 that insulator uses pin type and arrester is installed on all towers no matter what the discount rate is. With the increase of discount rate, LCC uniform annual value of the distribution system increases slightly.

(2) Effect of unit outage cost

Tab.7 Effect of outage cost on distribution system planning

\begin{tabular}{cccc}
\hline $\begin{array}{c}\text { Outage } \\
\text { cost } \\
(\mathrm{RMB})\end{array}$ & Type of insulator & Installation interval of arrester & $\begin{array}{c}\text { LCC(10,00 } \\
\text { 0 RMB) }\end{array}$ \\
\hline 2 & Glass insulator & One arrester every three spans. & 35.270 \\
4 & Pin insulator & Arrester is installed on all towers. & 45.776 \\
6 & Pin insulator & Arrester is installed on all towers. & 56.238 \\
8 & Glass insulator & Arrester is installed on all towers. & 66.711 \\
10 & Composite insulator & Arrester is installed on all towers. & 77.181 \\
\hline
\end{tabular}

It can be known from Table 7 that when outage cost is less than 2 RMB, distribution system planning of glass insulator and one arrester every three spans achieves the lowest LCC. When outage cost is higher than $2 \mathrm{RMB}$, the distribution system planning of pin insulator and arrester on all towers has the lowest LCC. With the increase of unit outage cost, the distribution system requires higher thunderstorm resistance capability. When the unit outage cost increases to $10 \mathrm{RMB}$, the minimum LCC is achieved by the distribution system planning of composite insulator and arrester on all towers. In other words, with the increase of unit outage cost, the total outage cost of the distribution system will increase significantly. To ensure the minimum outage cost, insulator with high insulating strength and arrester on all towers are needed.

\section{Conclusions}

This paper proposes a thunderstorm-resistance planning model of overhead distribution lines. It 
takes type of insulator and installation interval of arrester as the decision variables, and considers both reliability and economical efficiency. Combined with historical data, probability distribution models of lightning days and lightning current amplitude are established. By analyzing principle of lightning trip-out of lines, a long-term reliable model of overhead distribution line considering thunderstorm disaster is built. It enlists outage cost caused by lightning faults into the LCC of distribution system and gets the optimal thunderstorm-resistance planning through genetic algorithm by taking type of insulator and installation interval of arrester as decision variables.

The case study demonstrates that the proposed planning model could provide the optimal type of insulator and the optimal installation interval of arrester to distribution systems in different regions with different thunderstorm intensity. It has practical engineering significance to distribution system planning in regions with frequent thunderstorms.

\section{Acknowledgment}

This work is supported by the China Southern Power Grid Program (No. K-GZ2013-468).

\section{References}

[1] Feifei Zhang. The Method of Medium Voltage Distribution Network Planning Considering the Influence of Natural Disasters [D]. Beijing: North China Electric Power University, 2013.

[2] XU Guoxin, XIA Qing, KANG Chongqing. Research on Mode and Model of Anti-disaster Transmission Network Expansion Planning [J]. Automation of Electric Power Systems, 2010, 34(3): 17-21.

[3] DUAN Liming;LIU Nian;LIU Zongqi. A Lightning Vulnerability Evaluation Method in Medium Voltage Distribution Networks [J]. Modern Electric Power, 2014, 31(3): 61-67.

[4] CHEN Hao-yong, HU Bin, YE Rong. Transmission Network Planning Considering Weather Disaster [J]. Power System Technology, 2009, 33(20): 82-87.

[5] LIU Nian, MA Li, ZHU Tieming. Synthetical Assessment on Distribution Network Planning Scheme Considering Anti-Disaster Ability and Regional Characteristics [J]. Power System Technology, 2012(05): 219-225.

[6] ZHANG Fei-fei, LIU Nian, XIAO Rui, LIU Zong-qi. Planning of Medium-voltage Distribution Network Considering Wind Disaster Influence [J]. East China Electric Power, 2012, 40(07): 1178-1181.

[7] Baochu Yang, Xiaobo Liu, Songyu Dai. High Voltage Engineering [M]. Chongqing University Press, 2009.

[8] ZHANG Jianhua, ZENG Jianchao. Estimation of distribution algorithms using empirical distribution function as probability model [J]. Computer Engineering and Applications, 2011, 47(8): 33-35.

[9] Forbes C, ans M, Hastings N, et al. Statistical distributions[M]. John Wiley \& Sons, 2011. [10]CHEN Si-ming, TANG Jun, CHEN Xiao-ping. Calculation Analysis on Lightning Strike Trip-Out Rate of $10 \mathrm{kV}$ Distribution Line with Electric Geometry Method [J]. Insulators and Surge Arresters, 2013(004): 111-116.

[11]Qiangli Peng. Research on Intellectualized Diagnostic Device Applied to Distribution Lines [D]. Hunan: Changsha University of Science \& Technology ， 2011.

[12]Balijepalli N, Venkata S S, Richter Jr C W, et al. Distribution system reliability assessment due to lightning storms[J]. Power Delivery, IEEE Transactions on, 2005, 20(3): 2153-2159.

[13] Ying Lan . Distribution System Reliability Evaluation and Design Considering Influence of Typhoon [D]. Chongqing : Chongqing University , 2014.

[14]JinLiang He, Rong Zeng. Lightning Protection of Power Distribution Line [M]. Beijing: 
Tsinghua University Press, 2013.

[15]Jinliang H, Shanqiang G, Shuiming C, et al. Discussion on Measures Against Lightning Breakage of Covered Conductors on Distribution Lines[J]. IEEE Transactions on power Delivery, 2008, 23(2): 693-702.

[16]JIANG Zhou, HE Jinliang. Application of Surge Arrester Against Outage of Distribution Lines due to Breakages of Insulating Conductors [J]. Insulators and Surge Arresters, 2001(05): 32-35. [17]HOLLAND J H. Adaptation in natural and artificial systems: an introductiory analysis with applications to biology, control, and artificial intelligence[M] . 2nd ed. Cambridge: MIT Press, 1992.

[18]ZHENG Zhao_hui, ZHANG Yan, QIU Yu_huang. Genetic algorithm based on complex-valued encoding $[\mathrm{J}]$. Control Theory \& Applications, 2003, 20(1): 97-100.

[19]Allan R.N, Billinton R, Sjarief I, et al. A reliability test system for educational purposes basic distribution system data and results[J]. IEEE Transactions on Power Systems, 1991, 6(2): 813-820.

[20]Xiaobo Wang. Study on Life-Cycle-Cost Model and Its Application for Electrical Distribution Networks Considering the Reliability [D]. Chongqing: Chongqing University, 2008.

[21]http: //www. wunderground. com. /history/.

[22]http: //gis. ncdc. noaa. gov/map/viewer 